Volume 8, No. 7, July - August 2017

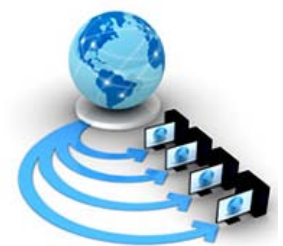

International Journal of Advanced Research in Computer Science

RESEARCH PAPER

\author{
Available Online at www.ijarcs.info
}

\title{
AN EFFICIENT CACHE-SUPPORT PATH COMPUTATION MODEL FOR ROAD MAPS
}

\author{
P. Praveen \\ Assistant Professor in CSE, \\ S R Engineering College, \\ Warangal,Telangana, India,
}

\author{
G. Mounika \\ M.Tech Student in CSE, \\ S R Engineering College, Warangal, \\ Telangana, India,
}

\author{
B.Rama \\ Assistant Professor in CS, \\ Kakatiya University, \\ Warangal,Telangana,India,
}

\begin{abstract}
In portable route administrations, on-street way arranging is an essential capacity that finds a course between a questioned begin area and a goal. While on streets, a way arranging inquiry might be issued because of dynamic considers different situations, for example, a sudden alter in driving course, surprising movement conditions, or lost of GPS signals. In these situations, way arranging should be conveyed in an opportune manner. The prerequisite of auspiciousness is considerably additionally difficult when a staggering number of way arranging questions is submitted to the server, e.g., during top hours. As the reaction time is basic to client fulfillment with individual route administrations, it is a command for the server to productively deal with the overwhelming workload of way arranging demands.To address this issue, we propose a system, to be specific, Path Planning by Caching (PPC), that intends to answer another way arranging inquiry proficiently by reserving and reusing generally questioned ways (questioned ways in short). Not at all like traditional cachebased way arranging systems where a cached question is returned just when it coordinates totally with another inquiry, PPC influences mostly coordinated questioned ways in cache to answer part(s) of the new question. Therefore, the server just needs to figure the unmatched way fragments, in this manner altogether decreasing the general system workload.
\end{abstract}

Keywords: Spatial Database, Path Planning, Cache.

\section{INTRODUCTION}

Because of advances in enormous information investigation, there is a developing requirement for versatile parallel calculations. These calculations envelop numerous areas including chart preparing, machine learning, and flag handling. Be that as it may, a standout amongst the most difficult calculations lie in chart preparing. Chart calculations are known to show low region, information reliance memory gets to, and high memory prerequisites.Indeed, even their parallel adaptations don't scale flawlessly, with bottlenecks coming from design imperatives, for example, cache impacts and on-chip organize movement. Way Planning calculations, for example, the well known Dijkstra's calculation, fall in the space of chart investigation, and show comparable issues. These calculations are given a diagram containing numerous vertices, with some neighboring vertices to guarantee availability, and are entrusted with finding the most limited way from a given source vertex to a goal vertex. Parallel executions dole out an arrangement of vertices or neighboring vertices to strings, contingent upon the parallelization system. These procedures normally present info reliance. Instability in choosing the ensuing vertex to hop to, results in low area for information gets to. In addition, strings focalizing onto the same neighboring vertex sequentialize systems because of synchronization and correspondence. Parceled information structures and shared factors ping-pong inside on-chip caches, bringing about lucidness bottlenecks. All these specified issues make parallel way arranging a test[7]. Earlier works have investigated parallel way arranging issues from different design points[3]. Way arranging calculations have been executed in chart structures. These circulated settings generally include vast bunches, and now and again littler groups of CPUs. Be that as it may, these works for the most part enhance workloads over various attachments and hubs, and for the most part constitute either entire shared memory or message passing (MPI) usage. On account of single hub (or single-chip) setup, a lot of work has been accomplished for GPUs are a couple of cases to give some examples. These works break down wellsprings of bottlenecks and examine approaches to relieve them. Summing up these works, we devise that most difficulties stay in the fine-grain inward circles of way arranging calculations. We trust that investigating and scaling way anticipating singlechip setup can limit the fine-grain bottlenecks. Since shared memory is productive at the equipment level, we continue with parallelization of the way arranging workload for singlechip multi-centers. The single-chip parallel usage can be scaled up at numerous hubs or groups granularity, which we talk about. Besides, programming dialect varieties for vast scale preparing likewise cause versatility issues that should be dissected successfully so far the most effective parallel 
shared memory executions for chart handling are in $\mathrm{C} / \mathrm{C}++$. In any case, because of security adventures and other potential vulnerabilities, other safe dialects are generally utilized as a part of mission-conveyed applications. Safe dialects ensure dynamic security watches that moderate vulnerabilities, and give simplicity of programming. In any case, security checks increment memory and execution overheads. Basic segments of code, for example, bolted information structures, now set aside greater opportunity to handle, and thus correspondence and synchronization overheads compound for parallel usage. Python is an unobtrusive case of a sheltered dialect, and thus we break down it's overheads with regards to our parallel way arranging workloads. This paper makes the accompanying commitments: ? We think about wellsprings of bottlenecks emerging in parallel way arranging workloads, for example, input reliance and adaptability, with regards to a solitary hub, single chip setup. ? We investigate issues emerging from safe dialects, for our situation Python, and talk about what safe dialects need to guarantee for consistent versatility. ? We plan to open source all described projects with the production of this paper[2].

\section{PATH PLANNING ALGORITHMS AND PARALLELIZATIONS}

Dijkstra that is an ideal calculation, is the accepted benchmark utilized as a part of way arranging applications. Notwithstanding, a few heuristic based varieties exist that exchange off parameters, for example, parallelism and exactness. ?-venturing is one case /

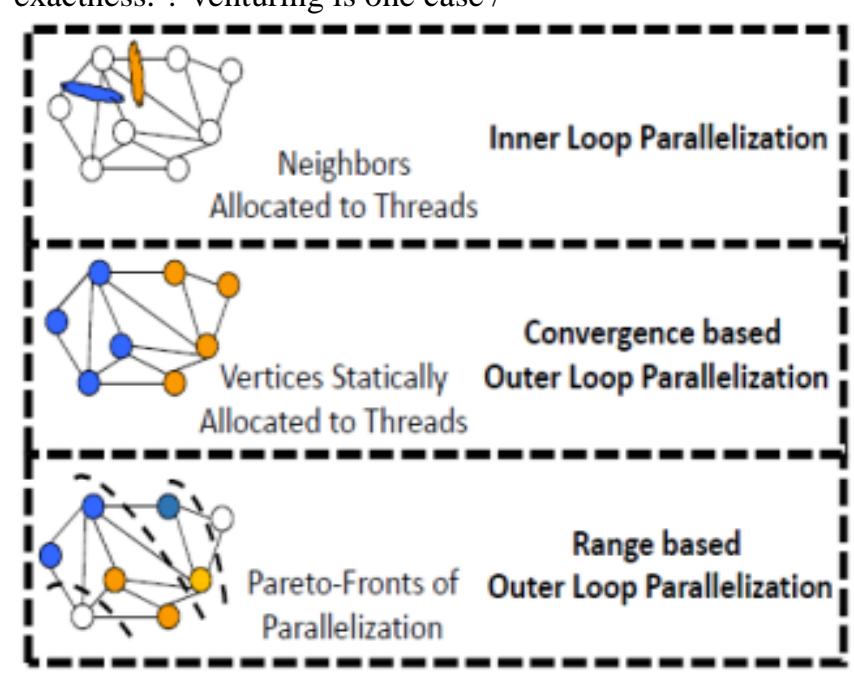

Fig.1. Dijkstra's Algorithm Parallelization's. Vertices Allocated to Threads Shown in Different Colors.

which orders diagram vertices and procedures them in various phases of the calculation[4]. The A*/D* calculations are another case that utilization forceful heuristics to prune out computational work (chart vertices), and just visit vertices that happen in the most limited way. With a specific end goal to keep up optimality and an appropriate gauge, we concentrate on Dijkstra's calculation in this paper.

\section{A. Dijkstra's Algorithm and Structure}

Dijkstra's calculation comprises of two fundamental circles, an external circle that navigates each chart vertex once, and an internal circle that crosses the neighboring vertices of the vertex chosen by the external circle. The most effective bland usage of Dijkstra's calculation uses a pile structure, and has an unpredictability of $\mathrm{O}(\mathrm{E}+\mathrm{V} \log \mathrm{V})$. In any case, in parallel executions, lines are utilized rather than piles, to diminish overheads related with re-adjusting the pile after each parallel cycle. Calculation 1 demonstrates the bland pseudo-code skeleton for Dijkstra's calculation.For every vertex, each neighboring vertex is gone to and contrasted and other neighboring vertices with regards to remove from the source vertex (the beginning vertex). The neighboring vertex with the base separation cost is chosen as the following best vertex for the following external circle emphasis. The separations from the source vertex to the neighboring vertices are then refreshed in the program information structures, after which the calculation rehashes for the following chose vertex.A bigger chart estimate implies more external circle emphasess, while a substantial diagram thickness implies more internal circle cycles. Therefore, these cycles convert into parallelism, with the diagram's size and thickness managing how much parallelism is exploitable. We talk about the parallelizations in ensuing subsections and show cases in Fig 1.

\begin{tabular}{|c|c|c|}
\hline \multicolumn{3}{|c|}{ Algorithm 1 Dijkstra's Algorithm Skeleton } \\
\hline \multicolumn{3}{|c|}{ 1: $\langle<<$ Initialize $D, Q\rangle>>$} \\
\hline 2 & for (Each vertex $u$ ) do & $\triangleright$ Outer Loop \\
\hline 3: & for (Each Edge of $u$ ) do & $\triangleright$ Inner Loop \\
\hline \multicolumn{3}{|c|}{ 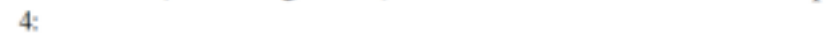 } \\
\hline 5: & \multicolumn{2}{|c|}{ 1. Calc. dist. from Current vertex to each neighbor } \\
\hline 6: & \multicolumn{2}{|c|}{ 2. Check for next best vertex $u$ among neighbors } \\
\hline
\end{tabular}

\section{B.Inner Loop Parallelization}

The inward circle in Algorithm 1 parallelizes the neighboring vertex checking. Each string is given an arrangement of neighboring vertices of the present vertex, and it registers a nearby least and updates that neighboring vertex's separation. An ace string is then called to take all the neighborhood essentials, and decrease to locate a worldwide least, which turns into the following best vertex to hop to in the following external circle emphasis. Boundaries are required between nearby least and worldwide least lessening ventures as the worldwide essentials must be figured when the ace string has admittance to all the neighborhood essentials. Parallelism is thusly reliant on the diagram thickness, i.e. the quantity of neighboring vertices per vertex. Meager charts constitute low thickness, and thusly can't scale with this sort of parallelization. Thick diagrams having high densities are relied upon to scale for this situation.

\section{Outer Loop Parallelization}

The external circle parallelization procedure parcels the diagram vertices among strings, portrayed in Algorithm 1. Each string runs inward circle emphasess over its vertices, and updates the separation clusters simultaneously. In any case, nuclear tickers over shared memory are required to refresh vertex separations, as vertices might share neighbors in various strings.

\section{Convergence Outer Loop Parallelization:}

The union based external circle statically allotments the chart vertices to strings. Strings chip away at their designated lumps autonomously, refresh speculative separation exhibits, and refresh the last separation cluster once each string finishes take a shot at its assigned vertices. The calculation then rehashes, until the last separation clusters balance out, where the adjustment sets the joining 
condition. Huge repetitive work is included as every vertex is registered upon various circumstances over the span of this present calculation's execution.

\section{Ranged based Outer Loop Parallelization:}

The range based external circle parallelization opens pare to fronts on vertices in every cycle. Vertices in these fronts are similarly partitioned among strings to register on, in any case, nuclear timekeepers are as yet required because of vertex sharing. As pare to fronts are brilliantly opened utilizing the diagram availability, a vertex can be securely casual only once throughout the calculation. Excess work is in this manner alleviated, while keeping up huge parallelism. Be that as it may, as beginning and last pare to fronts contain less vertices, restricted parallelism is accessible amid the underlying and last periods of the calculation. Higher parallelism is accessible amid the center periods of the calculation. This current calculation's accessible parallelism hereto takes after an ordinary appropriation, with time on the x-pivot.

\section{RELATED WORK}

An improved rendition [10] adds simple course bends to reduce vertices from being gone to and uses midway trees to lessen the pre-handling time. This work furthermore joins the upsides of the accomplish based and ATL approaches to manage diminish the amount of vertex visits and the interest space. The examination exhibits that the cross breed approach gives a transcendent result the extent that reducing question get ready time. Jung and Pramanik [11] propose the HiTi outline model to structure an enormous road sort out show. HiTi hopes to diminish the search space for the briefest way count. While HiTi fulfills unrivaled on road weight upgrades and diminishes stockpiling overheads, it achieves higher estimation costs when preparing the most short courses than the HEPV and the Hub Indexing methodologies [12], [3], [14]. To process time-subordinate brisk ways, Demiryurek et al. [5] propose the B-TDFP computation by using backward request to lessen the chase space. It gets a region level package plot which utilizes a road dynamic system to change each zone. In any case, a customer may slant toward a course with better driving information to the briefest way. Subsequently, Gonzalez et al. propose a flexible snappy way computation which utilizes speed and driving cases to improve the way of courses[2] [6]. The calculation uses a street various leveled segment and pre-calculation to upgrade the execution of the course figuring. The little road upgrade is a novel approach to manage improving the way of the course calculation. All together to improve the recuperation effectiveness of the way orchestrating structure, Thomsen et al. [1] propose another hold organization course of action to store the eventual outcomes of ceaseless inquiries for reuse later on. To redesign the hit extent, favorable position regard limit is used to score the routes from the question logs. In this way, the hit extent is extended, from now on lessening the execution times.In any case, the cost of building up a store is high, since the structure must figure the favorable position values for all sub-routes in a full-method for request comes about. For on-line, outline applications, setting up a generous number of simultaneous way inquiries is a basic issue. In this paper, we give another system to reusing the officially held request comes to fruition and an effective count for upgrading the question appraisal on the server.

\section{EXPERIMENTS}

\section{A. Dataset}

We lead a complete execution assessment of the proposed PPC system utilizing the street organize dataset of Seattle gotten from ACM SIGSPATIAL Cup 2012. The dataset has 25,604 hubs and 74,276 edges. For the inquiry log, we get the Points-of-intrigue (POIs) in Seattle from. Next, we arbitrarily select sets of hubs from these POIs as the source and goal hubs for way arranging questions. Four arrangements of question logs with various appropriations are utilized as a part of the trials: QLnormal and QLuniform are inquiry logs with ordinary and uniform disseminations, individually. QLcentral is utilized to reenact a substantial scale occasion (e.g., the Olympics or the World Cup) held in a city. QLdirection is to mimic conceivable driving conduct (e.g., altering course) in view of an irregular walk strategy depicted as takes after. We right off the bat arbitrarily create a question to be the underlying navigational course. Next, we arbitrarily attract a likelihood to decide the shot for a driver to alter course. The purpose of bearing change is dealt with as another source. This procedure is rehashed until the expected quantities of inquiries are produced. The parameters utilized as a part of our investigations are appeared in Table 2.

\section{B. Cache-Supported System Performance}

\section{Cache versus Non-Cache $T$}

he fundamental thought of a cache-upheld system is to use the cached inquiry results to answer another question. Subsequently, we are keen on discovering how much change our way arranging system accomplishes over a regular noncache system. We produce question sets of different sizes to look at the ways created by our PPC and A* calculation. The execution is assessed by two measurements:

a) Total number of went by hubs: it checks the quantity of hubs gone by a calculation under correlation in processing a way, and

b) Total inquiry time: it is the aggregate time a calculation takes to register the way.

As a matter of course, we apply 3,000 arbitrarily created questions to warm up the cache before continuing to quantify test comes about. Table 5 abridges the measurements of the over two measurements with five diverse estimated inquiry sets. From the insights we find that our cache-upheld calculation significantly lessens both the aggregate went by hubs and the aggregate inquiry time. By and large, PPC spares 23 percent of going to hubs and 30.22 percent of reaction time contrasted and a non-cache system.

TABLE II. Experimental Parameters

\begin{tabular}{lcc}
\hline Parameter & Default & Value \\
\hline Grid size & $2 \mathrm{~km}$ & $0.5 \sim 5 \mathrm{~km}$ \\
Cache size & $5 \mathrm{k}$ & $1 \mathrm{k} \sim 10 \mathrm{k}$ \\
\#Quries & $5 \mathrm{k}$ & $0.5 \mathrm{k} \sim 5 \mathrm{k}$ \\
Data sets & $Q L_{\text {mormal }}$ & $Q L_{\text {normal }}, Q L_{\text {wmiforms }}$ \\
& & $Q L_{\text {contral }}, Q L_{\text {dirnection }}$ \\
\hline
\end{tabular}


TABLE III. Performance Comparison between PPC and the Non-Cache Algorithm

\begin{tabular}{lcclcc}
\hline & \multicolumn{2}{c}{ HNodes } & & \multicolumn{2}{c}{ Time (ms) } \\
\cline { 2 - 3 } \cline { 5 - 6 } \#Query & PPC & $\mathrm{A}^{*}$ & & PPC & $\mathrm{A}^{*}$ \\
\hline $1 \mathrm{k}$ & 80,087 & 107,856 & & $14,190,670$ & $19,973,996$ \\
$2 \mathrm{k}$ & 157,162 & 215,459 & & $27,869,212$ & $39,889,166$ \\
$3 \mathrm{k}$ & 230,185 & 319,231 & & $41,493,092$ & $59,983,844$ \\
$4 \mathrm{k}$ & 328,879 & 419,345 & & $55,139,411$ & $79,937,684$ \\
$5 \mathrm{k}$ & 437,362 & 501,312 & & $69,843,232$ & $100,037,461$ \\
\hline
\end{tabular}

2. Cache with Different Mechanisms Performance Comparison:

We additionally analyze the execution of our system (PPC) with three other cache bolstered systems (LRU, LFU, SPC*) which embrace different cache substitution strategies or cache query arrangements. The initial two calculations identify ordinary (finish) cache hits when another question is embedded, yet refresh the cache substance utilizing either the Latest Recent Used calculation (signified as LRU) or the Least-Frequently Used substitution arrangements (LFU), separately. The third analyzed calculation, to be specific, the most brief way cache (SPC*), is a best in class cache bolstered system particularly intended for way arranging as PPC seems to be. SPC* additionally recognizes if any recorded questions in the cache coordinate the new inquiry superbly, yet it considers all subpaths in authentic question ways as chronicled inquiries also. We think about these four cache instruments by changing over the two measurements, number of went to hubs and reaction time, to sparing proportions against non-cache system for better introduction

$$
\begin{aligned}
& \delta_{\text {node }}=\text { nodes }_{\text {cache }} / \text { nodes }_{\text {nonca ce }} \times 100 \% \\
& \delta_{\text {time }}=\text { time }_{\text {cac he }} / \text { time }_{\text {noncac e }} \times 100 \%
\end{aligned}
$$

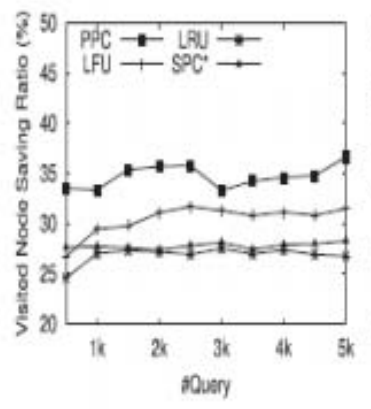

(a) $\delta_{\text {node }}$

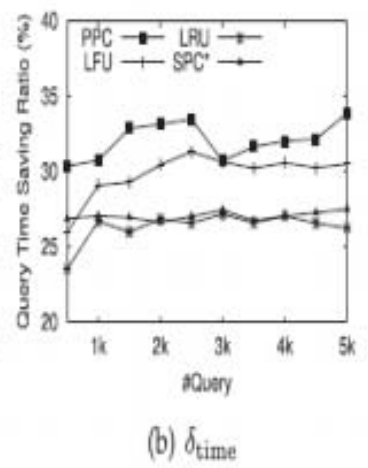

Fig.2. Execution Comparison with Four Cache Mechanisms in Terms of (a) Visited Node Saving Ratio, and (b) Query Time Saving Ratio with Different Numbers of Queries.

Gone to hub sparing proportion and Query efficient proportion show what number of hubs and how much time a calculation can spare from a non-cache steering calculation (e.g., $A^{*}$ ), separately. A bigger esteem demonstrates better execution. In the trial, we increment the aggregate question number from $1 \mathrm{k}$ to $5 \mathrm{k}$ and compute the over two measurements utilizing each cache component with the outcomes appeared in Fig.2. The $x$-hub speaks to the aggregate number of inquiries while the y-pivot demonstrates the metric values in rates. From these figures, we can see obviously that our cache strategy dependably accomplishes the best execution among all estimations. All things considered, LFU, LRU, SPC* and PPC visit 30.47 26.8627 .78 and 34.73 percent less hubs than $\mathrm{A}^{*}$ calculation, and lessens the computational time from $\mathrm{A}^{*}$ calculation by 29.83, 26.32, 27.04 and 32.09 percent, separately. As appeared, our calculation beats the other cache systems in way arranging altogether.

\section{Execution Analysis:}

In a cache-bolstered system, if the cached results can be (halfway) reused, the server workload can be reduced. In this manner, we measure the hit proportion as takes after: ??h ???? = ???????????? ??/|??| × $100 \%$ (4) where hits cache is the aggregate number of hits and $|\mathrm{Q}|$ is the aggregate number of inquiries. The hit proportion comes about utilizing distinctive cache instruments are looked at in Fig. 3, from which we find that, not surprisingly, PPC accomplishes a considerably higher hit proportion than the other three strategies in all situations. We additionally investigate the relationship between's the hit proportion and the execution measurements regarding went to hub sparing proportion and question efficient proportion with the outcomes appeared in Fig. 4. From the figures, we mention the accompanying objective facts: The went to hub sparing proportion and inquiry efficient proportion are corresponding to the hit proportion. By and large, with a higher hit proportion, the system execution enhances also. This is sensible as the system does not have to re-process the ways by breaking down the first street arrange chart, yet recovers the outcomes straightforwardly from the cache when a cache hit happens. In any case, sparing proportions for went to hubs are not the same with respect to the question time. For instance, PPC visits around 50 to 60 percent less hubs, yet the reaction time spared is around 30 to 40 percent. A conceivable reason is that diverse hubs assume distinctive parts in the guide. At the point when a question happens at sub diagrams with more intricate structures, the steering as a rule takes longer as its calculation may have a greater number of limitations than different hubs. The irregularity above is especially evident in PPC, presumably in light of the fact that PPC use incomplete hits to answer another inquiry. In any case, the rest of the sections still need the calculation from the street.

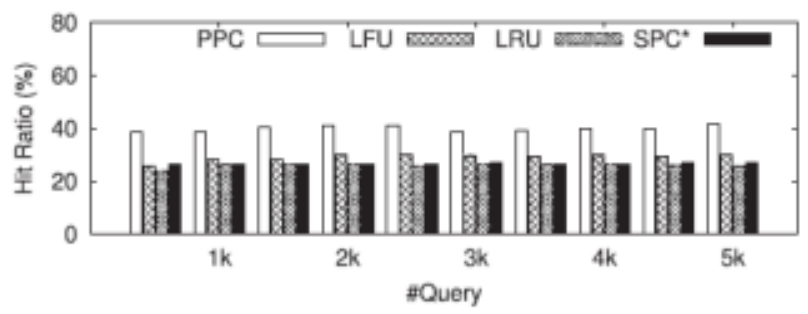

Fig.3. Performance Comparison with Four Cache Replacement Mechanisms in Terms of Hit Ratio.

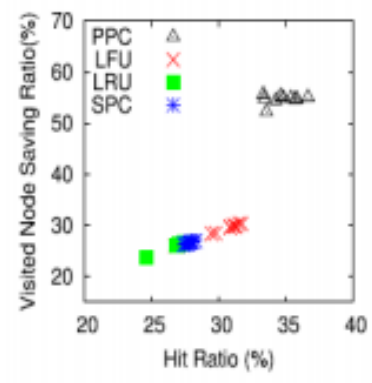

(a) $\delta_{\text {hit }}$ vs. $\delta_{\text {node }}$

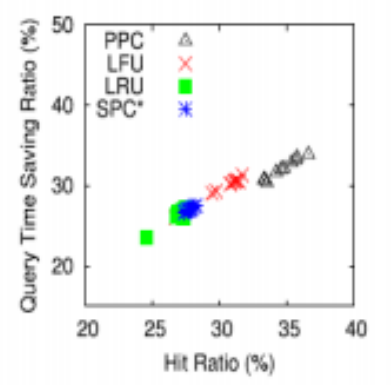

(b) $\delta_{\text {hit }}$ vs. $\delta_{\text {time }}$
Fig.4. Connection Between's (a) Hit Ratio and Visited Node Saving Ratio, and (b) Hit Ratio and Query Time Saving Ratio. 
organize chart, i.e., PPC does not generally spare sub ways in the event that they require complex calculations. Since PPC use both finish and halfway hit inquiries to answer another inquiry, we moreover measure the sparing hub proportion for incomplete hits. We ran a try different things with 5k questions, and have plotted the outcomes in Fig. 5. From the figure we can see that fractional hits show up uniformly along the fleeting measurement. By and large it accomplishes a 97.63 percent sparing proportion, which is very near the entire hit sparing proportion (100 percent). Among all cache hits, the take-up rates of finish and incomplete hits are represented in Fig. 6a and their sparing hub rate is appeared in Fig. 6b. The $\mathrm{x}$-hub is the question measure and the $y$-pivot is the rate values. See that incomplete hit does not accomplish a 100 percent sparing hub proportion. In any case, as incomplete hits happen a great deal more much of the time than finish hits, its general advantage to the system execution exceeds that of the entire hits. By and large, incomplete hits take up to 92.14 percent of the entire cache hit. The normal sparing hub proportion is 31.67 percent by fractional hits, 10 fold the amount of as that from finish hits at 3.04 percent.

\section{CONCLUSION}

We propose a system, in particular, Path Planning by Caching (PPC), that intends to answer another way arranging question effectively by storing and reusing truly questioned ways (questioned ways in short).The proposed system comprises of three primary segments: (i) PPattern Detection, (ii) Shortest Path Estimation, and (iii) Cache Management. iven a way arranging inquiry, which contains a source area and a goal area, PPC right off the bat decides and recovers various verifiable ways in cache, called PPatterns, that may coordinate this new question with high likelihood. The possibility of PPatterns depends on a perception that comparable beginning and goal hubs of two questions may bring about comparable most limited ways (known as the way rationality property). In the part PPatern Detection, we propose a novel probabilistic model to evaluate the probability for a cached questioned way to be helpful for noting the new inquiry by investigating their geospatial attributes. To encourage speedy discovery of PPatterns, rather than comprehensively examining all the questioned ways in cache, we plan a network based record for the PPattern Detection module. In light of these distinguished PPatterns, the Shortest Path Estimation module develops hopeful ways for the new inquiry and picks the best (most limited) one. In this segment, if a PPattern flawlessly coordinates the inquiry, we quickly return it to the client; generally, the server is made a request to figure the unmatched way fragments between the PPattern and the question. Since the unmatched sections are generally just a littler piece of the first inquiry, the server just procedures a "littler sub question", with a lessened workload. When we give back the evaluated way to the client, the Cache Management module is activated to figure out which questioned ways in cache ought to be expelled if the cache is full. An imperative piece of this module is another cache substitution arrangement which considers the one of a kind qualities of street systems. In this paper, we give another structure to reusing the beforehand cached inquiry comes about and in addition a compelling calculation for enhancing the question assessment on the server.

\section{REFERENCES}

[1] Ying Zhang, Member, IEEE, Yu-Ling Hsueh, Member, IEEE, Wang-Chien Lee, Member, IEEE, and Yi-Hao Jhang, "Productive Cache-Supported Path Planning on Roads", IEEE Transactions on Knowledge and Data Engineering, Vol. 28, No. 4, April 2016.

[2] Praveen, P., Ch Jayanth Babu, and B. Rama. "Big data environment for geospatial data analysis." Communication and Electronics Systems (ICCES), International Conference on. IEEE, 2016.

[3] H. Mahmud, A. M. Amin, M. E. Ali, and T. Hashem, "Shared execution of way questions on street systems," Clinical Orthopedics Related Res., vol. abs/1210.6746, 2012.

[4] L.Zammit, M.Attard, and K. Scerri, "Bayesian progressive displaying of movement stream - With application to Malta's street organize," in Proc. Int. IEEE Conf. Intell. Transp. Syst., 2013, pp. 1376-1381.

[5] Praveen, P., and B. Rama. "An empirical comparison of Clustering using hierarchical methods and K-means." Advances in Electrical, Electronics, Information, Communication and Bio-Informatics (AEEICB), 2016 2nd International Conference on. IEEE, 2016.

[6] S. Jung and S. Pramanik, "A productive way calculation show for progressively organized land guides," IEEE Trans. Knowl. Information Eng., vol. 14, no. 5, pp. 1029-1046, Sep. 2002.

[7] E. W. Dijkstra, "A note on two issues in connation with diagrams," Num. Math., vol. 1, no. 1, pp. 269-271, 1959.

[8] U. Zwick, "Correct and estimated removes in charts - a review," in Proc. ninth Annu. Eur. Symp. Calculations, 2001, vol. 2161, pp. 33-48.

[9] A. V. Goldberg and C. Silverstein, "Executions of Dijkstra's calculation in view of multi-level cans," Network Optimization, vol. 450, pp. 292-327, 1997.

[10] P. Hart, N. Nilsson, and B. Raphael, "A formal reason for the heuristic assurance of least cost ways," IEEE Trans. Syst. Sci. Cybern., vol. SSC-4, no. 2, pp. 100-107, Jul. 1968.

[11] A. V. Goldberg and C. Harrelson, "Registering the most limited way: A hunt meets diagram hypothesis," in Proc. ACM Symp. Discr. Calculations, 2005, pp. 156-165.

[12] R. Gutman, "Achieve based steering: another way to deal with most limited way calculations improved for street systems," in Proc. Workshop Algorithm Eng. Tests, 2004, pp. $100-111$.

[13] A. V. Goldberg, H. Kaplan, and R. F. Werneck, "Go after A*: Efficient indicate point briefest way calculations," in Proc. Workshop Algorithm Eng. Tests, 2006, pp. 129- 143.

[14] S. Jung and S. Pramanik, "An effective way calculation show for progressively organized land guides," IEEE Trans. Knowl. Information Eng., vol. 14, no. 5, pp. 1029-1046, Sep. 2002. 